\title{
Microfisicalismo y el alcance del argumento del zombi*
}

\section{Microphysicalism and the scope of the zombie argument}

\author{
Por: Reinaldo J. Bernal Velásquez \\ G.I. Problemas de filosofía \\ Facultad de Filosofía \\ Pontificia Universidad Javeriana \\ Bogotá, Colombia \\ E-mail: reinaldo-bernalv@javeriana.edu.co \\ ORCID: 0000-0001-6647-8582
}

Fecha de recepción: 23 de agosto de 2018

Fecha de aprobación: 18 de septiembre de 2018

Doi: 10.17533/udea.ef.n59a03

Resumen. El argumento del zombi, formulado por Chalmers (1996) contra la tesis según la cual la conciencia fenomenal es una propiedad de naturaleza fisica, supone que toda propiedad de un sistema fisico compuesto superviene (lógicamente) a partir de sus constituyentes fundamentales. En este texto, desarrollo en qué consiste esta suposición y muestro que la filosofía de la fisica brinda buenas razones para cuestionarla. En este orden de ideas, concluyo que el argumento del zombi no descarta una posición sobre la conciencia que la conciba como una propiedad física emergente, en el sentido de la emergencia-S (Howard, 2007). Termino discutiendo algunas objeciones.

Palabras clave: filosofia de la mente, argumento del zombi, conciencia fenomenal, fisicalismo, emergencia

Abstract. Chalmers'(1996) zombie argument against physicalism (or 'materialism') about (phenomenal) consciousness supposes that every property of a composed physical system supervenes (logically) on the system's fundamental constituents. In this paper, I discuss the significance of this supposition and I show that the philosophy of physics provides good grounds to resist it. As a result, I conclude that the zombie argument does not rule out a physicalist view of consciousness that conceives it as emergent in the sense of S-emergence (Howard, 2007). I finish by discussing some objections.

Keywords: philosophy of mind, zombie argument, phenomenal consciousness, physicalism, emergence

* Este texto se deriva principalmente de un trabajo de investigación desarrollado durante el año 2015 en el Institut Jean-Nicod (CNRS/ENS/EHESS), en París (Francia), bajo la tutoría del Profesor Uriah Kriegel, dentro del marco de la Beca Postdoctoral Fernand Braudel IFER incoming, otorgada al autor por la FMSH (Fondation maison des sciences de l'homme) en diciembre de 2014.

Cómo citar este artículo:

MLA: Bernal, Reinaldo. "Microfisicalismo y el alcance del argumento del zombi”. Estudios de Filosofía 59 (2019): 45-64.

APA: Bernal, R. (2019). Microfisicalismo y el alcance del argumento del zombi. Estudios de Filosofia, 59, 45-64.

Chicago: Reinaldo Bernal. "Microfisicalismo y el alcance del argumento del zombi". Estudios de Filosofía n. ${ }^{\circ} 59$ (2019): 45-64. 


\section{Introducción}

Chalmers (1996) presentó un argumento que sigue siendo muy influyente, llamado 'el argumento del zombi' [the zombie argument], para refutar el 'materialismo' en lo que se refiere, particularmente, a la conciencia fenomenal (Nagel, 1974; Block, 1995). ${ }^{1}$ Sostuvo que un mundo idéntico al mundo actual en todos los aspectos físicos, pero en donde no hay conciencia, es concebible. ${ }^{2}$ Sobre esta base, concluyó que en el mundo actual la conciencia no es una propiedad física. Escribió:

1. En nuestro mundo, hay experiencias conscientes.

2. Hay un mundo lógicamente posible físicamente idéntico al nuestro, en donde los hechos positivos sobre la conciencia de nuestro mundo están ausentes. ${ }^{3}$

3. Por lo tanto, los hechos sobre la conciencia son hechos adicionales de nuestro mundo, que van más allá de los hechos físicos. ${ }^{4}$

4. Luego, el materialismo es falso (Chalmers, 1996, p. 123, traducción propia).

Si efectivamente 'existe' un mundo $W^{*}$ que no difiere del mundo actual $W$ en absolutamente ningún respecto físico y en el cual, sin embargo, ninguna entidad tiene conciencia, se sigue claramente que la conciencia, en nuestro mundo, no es una propiedad física. La aceptación de este argumento depende entonces de la siguiente cuestión ¿es posible que en una réplica física exacta del mundo actual la conciencia esté completamente ausente? Para el fisicalista esto no es posible. Considera que la conciencia es una propiedad física y, por lo tanto, sostendría que una réplica exacta de $W$ que incluyera todos los aspectos físicos necesariamente

1 El argumento del zombi pertenece a la familia de 'argumentos de lo concebible' [conceivability arguments] o 'argumentos modales'. El primero fue presentado por Kripke (1980). Véase (Chalmers, 2010). No equiparo el término 'materialismo' con ‘fisicalismo' por razones que expondré en lo que sigue.

2 En adelante al utilizar 'conciencia' me referiré a la conciencia fenomenal.

3 Chalmers (1996) relaciona estrechamente la posibilidad lógica con la posibilidad metafísica: afirma que los mundos metafísicamente posibles y los mundos lógicamente posibles son los mismos. La diferencia entre estas posibilidades se ubica en el nivel epistemológico.

4 Chalmers $(2002 ; 2010)$ presenta el argumento bajo una forma que subraya la implicación desde lo concebible hacia lo posible, y argumenta a favor de la validez de esta implicación en los argumentos de los concebible. 
habría de incluir la conciencia. Sin embargo, esta sola afirmación constituiría una petición de principio frente al argumento de Chalmers teniendo en cuenta cómo él lo sostiene. Defiende la posibilidad del 'mundo zombi' $W^{*}$ con una línea de razonamiento basada en una tesis de 'superveniencia global':

$\mathrm{Si}$,

(premisa 1) Los hechos positivos sobre la conciencia no son hechos microfísicos. ${ }^{5}$

$\mathrm{Y}$,

(premisa 2) "Los hechos y las leyes de alto-nivel se siguen [con necesidad lógica] de todos los hechos microfísicos (quizás junto con las leyes microfísicas)" (Chalmers, 1996, p. 71, traducción propia).

Entonces,

(conclusión 1) Un mundo $W^{*}$ microfísicamente idéntico al mundo actual $W$ "tendría la misma estructura macroscópica que el nuestro, y la misma dinámica macroscópica" (Chalmers, 1996, p. 73, traducción propia).

Y sin embargo,

(conclusión 2) No habría experiencias conscientes en $W^{*}$.

La intuición central que subyace a este argumento es la siguiente: a menos de que adoptemos una forma de panpsiquismo, los hechos positivos sobre la conciencia no pueden seguirse de hechos microfísicos en virtud de una relación de necesidad metafisica ${ }^{6}$ Esto significa que a partir de un conjunto que contenga exclusivamente partículas físicas como, e.g., electrones y quarks, equipadas exclusivamente con propiedades físicas como, e.g., masa, carga eléctrica y espín, e independientemente de las relaciones espaciotemporales que pueda haber entre ellas, no es posible derivar bajo una relación de necesidad metafísica fenómeno alguno que involucre la conciencia. ${ }^{7}$

5 Esta premisa se traduce en la exclusión del panpsiquismo, que Chalmers discute en Chalmers (1996; $2002 ; 2010$ ). No descarta que una forma de panpsiquismo llamada 'monismo russelliano' pueda ser verdadera, y la introduce entonces como una alternativa a la falsedad del materialismo (Chalmers, 2010).

6 Esta intuición subyace con frecuencia a los argumentos a favor del panpsiquismo (Véase, e.g., Freeman, 2006).

7 Nótese que esta intuición supone que las propiedades físicas de las partículas no son ellas mismas propiedades fenomenales. Sostener que sí lo son corresponde a una versión del Monismo Russelliano (Véase Alter \& Nagasawa, 2015). 
El propósito del presente texto es controvertir el argumento de Chalmers. Para este fin, no voy a cuestionar la intuición central mencionada. ${ }^{8}$ En lugar de esto, voy a cuestionar la aceptación de la premisa 2 , que como veremos es un ingrediente esencial del 'microfisicalismo'. El rechazo de esta premisa conlleva, naturalmente, el rechazo del argumento. Supongamos que los hechos de alto nivel no se siguen con necesidad lógica de todos los hechos microfísicos, y que los hechos sobre la conciencia son hechos de alto nivel (i.e. que el panpsiquismo es falso). Si sucede que estos últimos son hechos físicos, como lo sostiene el fisicalismo, entonces no es cierto que un mundo $W^{*}$ microfísicamente idéntico al mundo actual $W$ tendría la misma estructura macroscópica y la misma dinámica macroscópica que $W$. La estructura y dinámica macroscópicas de $W^{*}$ serían diferentes de las de $W$ dada la ausencia en $W^{*}$ de los hechos positivos sobre la conciencia y en particular de todos los hechos macroscópicos que involucren interacciones entre la conciencia y propiedades de sistemas físicos; por ejemplo, el hecho de que un sujeto masajea su rodilla en la medida en que experimenta ahí un dolor.

Sin duda, en la filosofía de la mente el microfisicalismo sigue siendo la posición dominante entre los fisicalistas. En palabras de Papineau: "muchos [...] filósofos al parecer piensan en el fisicalismo como una suerte de compromiso con la primacía de lo microscópico. En su opinión, el fisicalismo no dice simplemente que todo es físico. También dice que todo está microscópicamente determinado" (2008, p. 126, traducción propia). Sin embargo, en la filosofía de la ciencia los argumentos que resultan en el rechazo del microfisicalismo tienen una historia relativamente larga. ${ }^{9}$ Naturalmente, Chalmers está al tanto de esta situación, pero respalda el microfisicalismo porque, en sus términos, es la posición ortodoxa en la actualidad (Chalmers, 2012, p. 293). Como veremos, este es un punto debatible y, en cualquier caso, el microfisicalismo está lejos de ser una posición inocente de amplia aceptación cuando se está discutiendo el argumento del zombi.

En las próximas secciones, primero, trazaré la distinción entre fisicalismo y microfisicalismo y, en ese orden de ideas, plantearé de manera precisa qué es aquello que está en juego a propósito de la premisa 2. En segundo lugar, mostraré que hay un caso muy significativo en la filosofía de la física que lleva a algunos filósofos influyentes a rechazar el microfisicalismo y, en esa medida, a favorecer una visión emergentista de la realidad física. En tercer lugar, concluiré que el argumento del

8 En Bernal (2012) argumento a favor de esta intuición.

9 Una discusión extensa sobre el microfisicalismo puede encontrarse en (Hüttemann, 2004). 
zombi no descarta una posición fisicalista emergentista sobre la conciencia. Para terminar, me ocuparé de algunas objeciones.

\section{Fisicalismo y microfisicalismo}

Como señalé previamente, la premisa 2 del argumento del zombi recoge una idea central del microfisicalismo. En esta sección, trazaré la distinción entre fisicalismo y microfisicalismo para mostrar que el primero no implica el segundo y, en esa medida, la premisa 2. Las formulaciones que voy a presentar de 'fisicalismo' y 'microfisicalismo' utilizan la relación de 'superveniencia'. ${ }^{10}$ Comenzaré entonces por introducir esta noción.

\subsection{Superveniencia}

Chalmers define esta relación, de manera canónica, como sigue: "Las propiedades-B supervienen sobre las propiedades-A si no hay dos situaciones [metafísicamente] posibles que sean idénticas con respecto a las propiedades-A mientras difieren con respecto a las propiedades-B" (Chalmers, 1996, p. 33, traducción propia). Las propiedades relacionadas pueden ser de cualquier tipo: físicas, geométricas, psicológicas, sociales, etc., y no es necesario que el tipo al que correspondan las propiedades-A coincida con el tipo al que correspondan las propiedades-B. Por ejemplo, cabe la posibilidad de que una propiedad estética supervenga sobre propiedades físicas.

Las propiedades-A constituyen la 'base de superveniencia' de las propiedades-B, y las primeras implican a las segundas con necesidad lógica o nomológica dependiendo de si el operador modal en 'situaciones posibles' cuantifica sobre mundos lógica o nomológicamente posibles respectivamente. Al

10 Las formulaciones del fisicalismo en términos de superveniencia (global) son utilizadas con mucha frecuencia, particularmente por Chalmers (e.g., Chalmers, 1996). No deja de ser cierto que estas formulaciones enfrentan ciertas dificultades. Primero, se ha sostenido que solo capturan el núcleo mínimo del fisicalismo (Lewis, 1983), o solo proveen condiciones necesarias mas no suficientes para su verdad. En segundo lugar, el camino para llegar a una definición precisa de 'superveniencia global' es intrincado. Leuenberger (2009) distingue entre tres relaciones que pueden llamarse apropiadamente 'superveniencia global' (una débil, una intermedia y una fuerte) y muestra que todas son problemáticas a pesar de su utilidad conceptual. 
utilizar 'superveniencia' sin especificación me refiero a la superveniencia lógica. ${ }^{11}$ La definición de Chalmers trata de propiedades, pero pueden darse definiciones análogas que relacionan otros tipos de elementos como entidades, estructuras, hechos, eventos y leyes. ${ }^{12}$ Utilizaré 'superveniencia' en este sentido más general. La superveniencia es una relación reflexiva, transitiva y no-simétrica. Chalmers la introduce con el propósito de desarrollar la premisa 2 y la conclusión 1 mencionadas.

Howard (2007) propone una formulación de 'superveniencia' específica para el caso en que los relata son estructuras matemáticas, que de acuerdo con el 'punto de vista semántico' sobre las teorías científicas son el vehículo mediante el cual se representan los sistemas físicos. ${ }^{13}$ Veremos en lo que sigue que, según el microfisicalismo, todas las propiedades de un sistema físico compuesto supervienen sobre las partículas básicas, junto con sus propiedades y trayectorias espaciotemporales, que lo integran. En esta medida, la formulación de Howard resulta particularmente útil para especificar lo que sostiene el microfisicalismo. He aquí dicha formulación:

\begin{abstract}
La superveniencia es una relación óntica entre estructuras. Una estructura, $S_{x}$, es un conjunto de entidades, $E_{x}$, junto con sus propiedades y relaciones, $P R_{\mathrm{x}}$. Una estructura, $S_{B}$, característica de un nivel, $B$, superviene sobre una estructura, $S_{A}$, característica de otro nivel, $A$, si y solo si las entidades de $S_{B}$ están compuestas por las entidades de $S_{A}$ y las propiedades y relaciones, $P R_{B}$, de $S_{B}$ están completamente determinadas por las propiedades y relaciones, $P R_{A}$, de $S_{A}$. Una forma de entender el sentido relevante de 'determinación' es requiriendo que no haya diferencias en el nivel $B$, por ejemplo diferentes valores de un parámetro como la temperatura de un gas, sin que haya una diferencia correspondiente en el nivel $A$, por ejemplo la energía cinética promedio de las moléculas que constituyen el gas (p. 143, traducción propia).
\end{abstract}

El siguiente caso, propio de la geometría, ilustra de manera simple la relación de superveniencia. Considérense tres puntos en el plano cartesiano, A, B y C, ubicados en las siguientes coordenadas: A $(0,0)$; B $(4,0)$; C $(0,3)$. Considérense ahora los siguientes tres segmentos que unen pares de estos puntos: $[\mathrm{AB}],[\mathrm{AC}] \mathrm{y}$

11 La superveniencia nomológica debe distinguirse de la emergencia de superveniencia-nomológica (Van Cleve, 1990; McLaughlin, 1997). En la segunda, debe haber una ley fundamental de la naturaleza conectando la base de superveniencia/emergencia con la propiedad superveniente/emergente. En cambio, la superveniencia nomológica admite una relación de implicación metafísica como aquello que realiza esta conexión.

12 Sobre definiciones de superveniencia (véase, e.g., McLaughlin, 1995).

13 Véase, e.g., Suppes (2002). 
$[\mathrm{BC}]$. El triángulo $\mathrm{ABC}$, con sus propiedades, superviene sobre estos tres segmentos, con sus propiedades. En primer lugar, nótese que la presencia del triángulo en el plano está garantizada por la presencia de esos tres segmentos. Una vez los tres segmentos están presentes en las ubicaciones señaladas, el triángulo también está presente; no es necesario nada adicional a los tres segmentos. En segundo lugar, nótese que todas las propiedades geométricas del triángulo están determinadas por las propiedades geométricas de los segmentos. Dadas las orientaciones y longitudes de los segmentos, el triángulo tiene, entre otras, la propiedad de ser un triángulo rectángulo. En esta medida, nótese que no es posible modificar propiedades del triángulo, como que tenga un área de 6 unidades, sin modificar propiedades de los segmentos.

\subsection{Fisicalismo}

El fisicalismo puede formularse como la tesis según la cual todas las entidades que habitan el mundo actual, sus propiedades, y todos los hechos y eventos que las involucran, tienen una naturaleza física en el siguiente sentido:

(naturaleza fisica) Un elemento tiene una naturaleza física si superviene sobre un conjunto de elementos físicos.

Aquí, 'elementos físicos' incluye entidades físicas, sus propiedades, y sus historias espacio-temporales. Claramente, si el fisicalismo es verdadero, la totalidad de elementos físicos de nuestro mundo constituye una base de superveniencia suficiente para todo lo que hay en él, incluyendo la realidad social. ${ }^{14}$

No intentaré ofrecer una propuesta sobre qué es para un elemento ser físico. Esto exigiría un extenso interludio con pocas probabilidades de éxito. En efecto, se enfrenta en este punto el Dilema de Hempel: o bien tomamos como 'físico' aquello que propone en su ontología la física actual, o bien aquello que propondría una física ideal, i.e., correcta y completa. En el primer caso se subordina la categoría de 'lo físico' a la contingencia histórica de las teorías, y en el segundo caso renunciamos a la posibilidad de establecer qué es 'lo físico' ya que no contamos con la física ideal. ${ }^{15}$ Sin embargo, a pesar de este dilema, parece indiscutible que tenemos una

14 Véase Searle (1995).

15 Un intento interesante por especificar lo 'físico' evitando caer en el Dilema de Hempel se encuentra en Papineau (2008). 
noción intuitiva de 'lo físico' que se apoya en casos paradigmáticos y que se utiliza recurrentemente en los debates filosóficos. Es claro que un protón, una molécula y la carga eléctrica son elementos físicos. En contraste, los billetes y los precios no lo son, aunque tengan una 'naturaleza física' si el fisicalismo es verdadero. En efecto, lo que hace que un objeto que denominamos 'billete' sea efectivamente un billete no son sus propiedades físicas — ser un papel rectangular con tintasino la función social que dicho objeto cumple, si bien esas propiedades se han seleccionado buscando que el objeto pueda cumplir adecuadamente su función. Nótese que cambios institucionales, como sucedió con la implementación del Euro en Europa, pueden llevar a que un objeto pierda su estatus de billete, i.e., pierda su poder transaccional, sin que tenga lugar ninguna alteración en sus propiedades físicas. Como señalé anteriormente, no ofreceré una propuesta sobre qué es para un elemento 'ser físico'. Sin embargo, abordaré más adelante (\$4) un aspecto de la cuestión. Sostendré que es razonable clasificar como física a una propiedad $N$ en la medida en que esté relacionada nomológicamente con propiedades paradigmáticamente físicas.

\subsection{Microfisicalismo}

De forma similar al fisicalismo, el microfisicalismo puede formularse como la tesis según la cual todas las entidades que hay en nuestro mundo, sus propiedades, y todos los hechos y eventos que las involucran, tienen una naturaleza microfísica en el siguiente sentido: ${ }^{16}$

(naturaleza microfísica) Un elemento tiene una naturaleza microfísica si superviene sobre un conjunto de elementos físicos básicos.

Aquí, 'elementos físicos básicos' incluye exclusivamente partículas simples, sus propiedades y sus historias espacio-temporales. Claramente, si el microfisicalismo es verdadero la totalidad de elementos físicos básicos de nuestro mundo constituye una base de superveniencia suficiente para la totalidad de lo que hay en él, incluyendo, además de los constructos sociales, los sistemas físicos compuestos con sus propiedades y relaciones (de alto-nivel). Dado que, como vimos, los

16 Para Hüttemann (2004), el microfisicalismo puede articularse por lo menos de tres maneras: como una tesis de 'micro-determinación' (que involucra propiedades), como una tesis de 'micro-gobierno' (que involucra leyes) y como una tesis de 'micro-causación'. Con la ayuda de pocos puntos plausibles es posible derivar estas tres tesis de la formulación en términos de superveniencia que propongo. 
elementos supervenientes se siguen de su base de superveniencia con necesidad lógica, es claro que la premisa 2 _ "Los hechos y las leyes de alto-nivel se siguen [con necesidad lógica] de todos los hechos microfísicos (quizás junto con las leyes microfísicas)"- recoge un punto central del microfisicalismo.

Nótese que la realidad microfísica sobre la cual, de acuerdo con el microfisicalismo, todo superviene, no debe concebirse como involucrando elementos que no pertenezcan al nivel más básico de la ontología física. De lo contrario, el microfisicalismo perdería lo que lo hace atractivo en primer lugar: la idea de que todo puede reducirse ontológicamente en términos de entidades simples, con sus propiedades (básicas) y relaciones, sin residuo. Es más: si 'lo microfísico' incluyera elementos que fueran más allá de los básicos, una distinción clara entre este nivel fundamental y niveles superiores difícilmente podría trazarse y justificarse.

El microfisicalismo es intuitivamente claro y tiene la ventaja de proponer una ontología austera. Imagínese que debemos crear un inventario exhaustivo de todo lo que ha existido y ocurrido en el mundo actual. Si el microfisicalismo es verdadero, cuando incluimos todos los elementos básicos el inventario queda completo. Sin duda es útil para efectos epistemológicos incluir elementos que no son básicos, pero estos serían redundantes desde el punto de vista ontológico.

Ciertamente, el microfisicalismo es atractivo y puede fácilmente tomarse como una tesis central de las ciencias naturales contemporáneas. De hecho, para Chalmers $(1996 ; 2010)$ el monismo materialista involucra un compromiso con el microfisicalismo. Por ejemplo, escribe: "Hay un amplio acuerdo sobre que el materialismo requiere que $P$ implique necesariamente todas las verdades", donde " $P[\mathrm{es}]$ el conjunto de todas las verdades microfísicas sobre el universo" (Chalmers, 2010 , p. 110, traducción propia). Sin embargo, veremos en la próxima sección que las física contemporánea avala la posibilidad de que el microfisicalismo sea falso. Pero, ¿implica el fisicalismo al microfisicalismo o, a la inversa, implica el microfisicalismo al fisicalismo?

Imagínese un mundo $W^{*}$ definido como una réplica física mínima exacta del mundo actual $W$ : Toda entidad física de $W$, con sus propiedades e historia, tiene una contraparte cualitativamente idéntica en $W^{*}$ y no hay nada más en $W^{*}$. Si el fisicalismo es verdadero, entonces el que $W^{*}$ sea una réplica física mínima exacta de $W$ conlleva que sea una réplica simpliciter: $W$ es idéntico a $W^{*}$. Todos los organismos, personas, instituciones, eventos, etc., de $W$, tienen contraparte en $W^{*}$ 
y viceversa. Por el contrario, si el fisicalismo es falso, entonces $W$ y $W^{*}$ difieren: algunas entidades de $W$ carecen de una contraparte en $W^{*}$.

A manera de ejemplo, consideremos el siguiente escenario: las propiedades mentales supervienen sobre elementos físicos. Si el fisicalismo es verdadero, en una réplica física mínima exacta de nuestro mundo también se encuentran las propiedades mentales (y todo lo demás que haya en nuestro mundo). Ahora, consideremos el escenario opuesto: las propiedades mentales no tienen una naturaleza física; la mente es, por ejemplo, un alma cartesiana. En este caso, una réplica física mínima exacta de nuestro mundo difiere de él: no contiene, en particular, propiedades mentales.

De manera similar a lo anterior, imagínese un mundo $W^{* *}$ definido como una réplica microfísica mínima exacta del mundo actual $W$ : Todas las entidades físicas básicas de $W$, con sus propiedades e historia, tienen contrapartes cualitativamente idénticas en $W^{* *}$. Si el microfisicalismo es verdadero, entonces el que $W^{* *}$ sea una réplica microfísica mínima exacta de $W$ conlleva a que sea una réplica simpliciter: $W$ es idéntico a $W^{* *}$. Todas las personas, instituciones, etc. de $W$ y, en particular, todos los sistemas físicos compuestos de $W$ junto con sus propiedades (e.g. los objetos macroscópicos rígidos que tienen determinada temperatura), tienen contraparte en $W^{* *}$ y viceversa. Esto último es así en la medida en que, de acuerdo con el microfisicalismo, los elementos físicos que no son básicos supervienen (al igual que todo lo demás presente en $W$ ) sobre elementos físicos básicos.

Por el contrario, si el microfisicalismo es falso, $W$ y $W^{* *}$ difieren, y nótese que pueden diferir de dos maneras. Por un lado, y de manera análoga con el caso en que el fisicalismo es falso, $W$ y $W^{* *}$ pueden diferir a propósito de aquellas entidades presentes en $W$ que no supervengan sobre elementos físicos. $\mathrm{Si}$, por ejemplo, las propiedades mentales no tienen una naturaleza física, estas no estarán presentes en $W^{* *}$. Pero, por otro lado, a diferencia del caso en que el fisicalismo es falso, $W$ y $W^{* *}$ también pueden diferir en respectos físicos: algunas entidades físicas compuestas de $W$ pueden carecer de una contraparte idéntica en $W^{* *}$. Este es el caso si algunos elementos físicos presentes en $W$ no supervienen sobre elementos físicos básicos. $\mathrm{Si}$, por ejemplo, las propiedades mentales son físicas pero no supervienen sobre elementos físicos básicos, una réplica microfísica mínima exacta de nuestro mundo no contiene propiedades mentales. 
A partir de lo anterior, queda claro que la verdad del microfisicalismo implica la verdad del fisicalismo: si el microfisicalismo es verdadero, una réplica microfísica mínima exacta de $W$ también es una réplica física mínima exacta de $W$. $W^{* *}$ es idéntico a $W^{*}$ y los dos son a la vez idénticos a $W$. Esto es así en la medida en que todo lo que existe en $W$, ya sean elementos físicos que no son básicos, o elementos que no son físicos (pero tienen una naturaleza microfísica), superviene sobre elementos físicos fundamentales.

También queda claro que, por el contrario, la verdad del fisicalismo no implica la verdad del microfisicalismo: que una réplica microfísica de $W$ sea también una réplica física de $W$ no es una aseveración verdadera a priori. Es posible que los mundos $W^{*}$ y $W^{* *}$ difieran: puede haber propiedades en el mundo actual $W$ que estén replicadas (por definición) en $W^{*}$ pero no (por cuestiones empíricas) en $W^{* *}$. Este es el caso si en el mundo actual $W$ existen propiedades físicas de sistemas físicos compuestos que no supervienen exclusivamente sobre elementos físicos básicos.

\section{El microfisicalismo cuestionado}

Dentro de un marco fisicalista, rechazar el microfisicalismo es, como vimos, sostener que hay propiedades físicas de sistemas físicos compuestos que no supervienen sobre elementos físicos básicos. Argumentos en esta dirección tienen una historia relativamente larga en la filosofía de la ciencia. Corresponden a algunas teorías de la emergencia que, siguiendo a Howard (2007), pueden agruparse como 'emergencia-S' [S-emergence], dado que él define la emergencia-S simplemente como la violación de la superveniencia. ${ }^{17}$ Las formulaciones de teorías de la emergencia-S de mayor aceptación se encuentran en Van Cleve (1990), O'Connor (1994), McLaughlin (1997) y Kim (1999).$^{18}$ La más influyente es la 'emergencia de superveniencia nomológica' [nomological-supervenience emergence] (Van Cleve, 1990; McLaughlin, 1997), que McLaughlin (1997) define como sigue:

Si $P$ es una propiedad de $w$, entonces $P$ es emergente si y solo si (1) $P$ superviene con necesidad nomológica, pero no con necesidad lógica, sobre propiedades que las partes de $w$ tienen tomadas separadamente o en otras combinaciones; $\mathrm{y}(2)$ algunos de los principios

17 En primer lugar, Howard (2007) argumenta que no hay una relación directa entre la reducción y la superveniencia. Luego, define la 'emergencia-R' [R-emergence] como la violación de la reducción y la emergencia-S como la violación de la superveniencia.

18 Si bien Kim (1999) formula una teoría de emergencia-S él no cree en la veracidad de esta teoría y la cuestiona. McLaughlin (1997), por su parte, no se compromete con la veracidad de la teoría de emergencia-S que formula, pero tampoco la descarta. 
de superveniencia que conectan propiedades de las partes de $w$ con que $w$ tenga $P$ son leyes fundamentales" (p. 39, traducción propia).

Las "propiedades que las partes de $w$ tienen tomadas separadamente o en otras combinaciones" constituyen la 'base de emergencia' de P.

Las teorías de emergencia-S son ontológicas y sincrónicas. Son ontológicas en el siguiente sentido: las propiedades emergentes se conciben como algo que va más allá de las bases de emergencia correspondientes. Si bien su existencia depende de la existencia de las bases, son constituyentes adicionales de la realidad. Las teorías ontológicas, en general, están motivadas por la intuición de que, si bien es seguro que existen relaciones estrechas entre las propiedades de alto-nivel y propiedades básicas, y si bien podemos entender mejor los niveles altos con modelos de niveles bajos, algunas propiedades de alto-nivel — las emergentes - son (al menos) tan reales como se considera que son las básicas. ${ }^{19}$

Las teorías de emergencia-S son sincrónicas porque las propiedades emergentes se conciben como coexistiendo con las bases de emergencia correspondientes. Nótese que las relaciones de emergencia sincrónicas no pueden ser causales (por lo menos bajo posiciones ortodoxas, donde las causas siempre preceden los efectos) en contraste con las relaciones de emergencia diacrónicas. ${ }^{20}$

Como vimos, si existen propiedades emergentes en la naturaleza la premisa 2 del argumento del zombi no se sostiene. Ahora bien, hay un fenómeno físico que ha sido particularmente fructífero para sostener argumentos a favor de la existencia de propiedades emergentes-S: el entrelazamiento cuántico. Se dice de dos sistemas cuánticos que están entrelazados cuando el estado del uno no puede describirse sin referirse al estado del otro, es decir, cuando sus estados no pueden especificarse de manera independiente. Los argumentos a favor de la existencia de propiedades emergentes-S basados en el entrelazamiento cuántico incluyen, entre otros, Teller (1986), Esfeld (2004) y Howard (2007). ${ }^{21}$ La idea central es que siempre que dos sistemas cuánticos, $a$ y $b$, se entrelazan, el sistema entrelazado resultante $S$ instancia

19 De acuerdo con Hüttemann (2004), el atractivo del microfisicalismo se deriva precisamente del éxito de las micro-explicaciones.

20 El emergentismo no siempre es concebido como una forma de fisicalismo. De hecho, algunos autores, e.g., Nida-Rümelin (2007), proponen teorías emergentistas como alternativas dualistas.

21 Humphreys (2008) se encuentra entre los argumentos a favor de la existencia de propiedades emergentes más influyentes basados en el entrelazamiento cuántico. Sin embargo, este argumento concierne una forma de emergencia diacrónica en contraste con la emergencia-S. 
propiedades que no supervienen sobre propiedades intrínsecas de $a$ y de $b$ tomados separadamente..$^{22}$ Teller (1986) escribe:

Pero dadas estas dos propiedades compuestas, la mecánica cuántica nos dice que su superposición, $w_{1}^{a} w_{2}^{b}+w_{2}^{a} w_{1}^{b}$, es también una propiedad que el par, $a, b$, puede tener. Esta propiedad es una propiedad relacional que se da entre $a$ y $b$, o para $a$ y $b$ de manera colectiva; y a excepción de casos degenerados esta propiedad no se reduce a y no superviene sobre propiedades no-relacionales de a y $b$ tomados separadamente (p. 78-79, itálicas añadidas, traducción propia).

\section{De manera similar, Esfeld (2004) escribe:}

Los casos de entrelazamiento cuántico son casos de no-separabilidad en la medida en que únicamente hay un estado compuesto que determina ciertas correlaciones que se dan entre los sistemas en cuestión sin que sea posible atribuir estados significativos (o propiedades dependientes-de-estados si de eso se trata) a cada uno de los estados en cuestión [...] Se puede concebir el estado compuesto de un todo cuántico como incluyendo propiedades intrínsecas del todo, esto es, propiedades intrínsecas que no supervienen sobre propiedades intrínsecas de las partes (p. 609-610, itálicas añadidas, traducción propia).

Howard (2007) explícitamente sostiene que las propiedades nosupervenientes de los sistemas cuánticos entrelazados son muy buenos candidatos para ser propiedades emergentes-S:

Cómo y por qué las propiedades de un par de sistemas cuánticos que han interactuado previamente y que, por lo tanto, están entrelazados, no cumplen con supervenir sobre las propiedades de los dos sistemas tomados de manera individual se entiende perfectamente bien y hoy en día se demuestra recurrentemente en el laboratorio, como en experimentos que ponen a prueba el teorema de Bell [...] En mi opinión, las correlaciones cuánticas características de los estados compuestos entrelazados ofrecen mejores razones para la atribución del estatus de propiedades emergentes que cualquiera de las otras propiedades presentes en la naturaleza que han sido nominadas para el premio (p. 150, itálicas añadidas, traducción propia).

Sin lugar a dudas, la interpretación de la mecánica cuántica es una cuestión abierta muy debatida. En particular, no es posible probar que el microfisicalismo es definitivamente falso apelando al entrelazamiento cuántico (y de hecho a ningún fenómeno físico). El microfisicalismo es una tesis metafísica y toda teoría científica, dado su carácter empírico, puede ser falsa a pesar del nivel de aceptación que pueda llegar a alcanzar. Sin embargo, el microfisicalismo está motivado por la aceptación

22 En Bernal (2016) explico detalladamente en qué consiste el fenómeno de entrelazamiento cuántico para un sistema EPR y, en la línea de los autores citados, argumento a partir del Teorema de Bell que el entrelazamiento cuántico involucra propiedades emergentes-S. Este texto pretende ser accesible para lectores que no cuenten con un conocimiento técnico de la mecánica cuántica. 
de teorías contemporáneas en las ciencias naturales, y entonces es legítimo cuestionarlo sobre la base de la física contemporánea. Mi punto es simplemente que el microfisicalismo no puede tomarse como algo dado en el contexto presente.

Bajo la hipótesis de que existen en la naturaleza propiedades emergentes-S, como las tendrían los sistemas de entrelazamiento cuántico, la realidad material se concibe como una estructura multinivel. En el nivel más bajo se encuentran los elementos físicos básicos, y en niveles superiores, ya sean físicos, químicos, o biológicos, se encuentran propiedades ontológicamente nuevas con respecto al nivel más bajo. Ciertamente, la existencia de estas propiedades está subordinada al nivel más bajo. En este sentido, los elementos físicos básicos son una condición necesaria para la instanciación de propiedades emergentes-S. Sin embargo, en la medida en que estas propiedades no supervienen sobre el nivel más bajo, los elementos físicos básicos no son una condición suficiente para su instanciación. Ahora bien, desde el punto de vista epistemológico la existencia de propiedades emergentes-S no va en contravía de la explicación analítica: las propiedades de un sistema pueden explicarse a partir de las propiedades de sus partes y de cómo estas se relacionan entre sí. Sin embargo, para que esta explicación sea exitosa, es preciso incorporar leyes de emergencia-S.

\section{Objeciones y respuestas}

En las secciones precedentes cuestioné la validez del argumento del zombi argumentando que hay buenas razones para considerar que el microfisicalismo, y con él la afirmación de que "los hechos y las leyes de alto-nivel se siguen [con necesidad lógica] de todos los hechos microfísicos", son erróneos. Sugerí la aceptación de una visión emergentista de la naturaleza, en el sentido de la emergencia-S.

Ciertamente, no he dado razones para considerar que la conciencia, en particular, sea una propiedad emergente-S. Del hecho (si es un hecho) de que sistemas cuánticos relativamente simples tengan propiedades emergentes-S, nada parece seguirse a propósito de la naturaleza de la conciencia. En principio, solo organismos biológicos extremadamente complicados tienen conciencia, y además la conciencia suscita preguntas filosóficas específicas. Sin embargo, nótese que el hecho (si es un hecho) de que sistemas cuánticos relativamente simples instancien propiedades emergentes-S exige una ontología no-microfisicalista del mundo natural, lo cual abre la posibilidad de que haya propiedades emergentes más allá 
de los sistemas microfísicos. ${ }^{23}$ Ahora bien, hay razones independientes de cualquier consideración sobre la mecánica cuántica para sostener que la conciencia es una propiedad física emergente. ${ }^{24} \mathrm{Si}$ bien desarrollar este punto sobrepasa el propósito y límite del texto presente, permítaseme señalar lo siguiente: Si (1) adoptamos una posición realista frente a la conciencia, (2) rechazamos el panpsiquismo, i.e., la idea de que partículas simples instancian propiedades fenomenales, (3) aceptamos que las propiedades fenomenales no supervienen sobre elementos físicos básicos, y (4) consideramos que el fisicalismo es verdadero, no parece quedar otro camino que sostener que la conciencia es una propiedad emergente-S. ${ }^{25}$ Para terminar, voy a ocuparme brevemente de cinco objeciones frente a la posición sugerida, a saber, que la conciencia es una propiedad emergente-S.

Objeción 1: La afirmación según la cual la conciencia podría ser una propiedad emergente-S coincide con el 'dualismo naturalista' de Chalmers: él sostiene, precisamente, que la conciencia superviene nomológicamente, pero no metafísicamente, sobre lo microfísico.

Respuesta: Dice Chalmers cuando presenta el dualismo naturalista: "Es entonces más natural considerar la experiencia como una propiedad fundamental que no es una propiedad física, y considerar las leyes psicofísicas como leyes fundamentales de la naturaleza que no son leyes de la física" (1996, p. 128-129, traducción propia). Es claro entonces que para Chalmers las leyes psicofísicas que estarían involucradas en la superveniencia nomológica de la conciencia relacionan algo físico con algo que no es físico (en concordancia con el argumento del zombi). Esto contrasta con la concepción de la emergencia presentada - la emergencia-Sen la cual las propiedades emergentes (como el entrelazamiento cuántico) son físicas.

Desarrollo de la objeción 1: Supóngase que hay leyes psicofísicas en virtud de las cuales la conciencia emerge en el sentido de la emergencia-S. Persiste aún la posibilidad de que la conciencia no sea una propiedad física. En efecto, es arbitrario tomar las leyes psicofísicas como 'leyes de la física' y, de esta manera, afirmar que la conciencia es física, en lugar de tomar estas leyes - siguiendo a Chalmerssimplemente como leyes fundamentales de la naturaleza que no suponen que la conciencia sea física.

23 De hecho, la mecánica cuántica no restringe el entrelazamiento a sistemas microscópicos compuestos por unas pocas partículas. En principio, cualesquiera sistemas físicos interactuando se entrelazan.

24 Sobre emergencia en la filosofía de la mente (véase MacDonald \& MacDonald, 2010).

25 En Bernal (2012) desarrollo este argumento. 
Respuesta: No estoy seguro de cómo entender en qué sentido una ley de la naturaleza podría relacionar algo físico con algo no-físico. Estas leyes son, mínimamente, sentencias que capturan regularidades en la naturaleza. Ahora bien, si hay una regularidad que involucra un elemento físico paradigmático $M$ y un elemento $N$, esto parece ser suficiente para que $N$ caiga bajo la categoría de lo que tiene una naturaleza física. ¿Por qué pensamos, e.g., que las ondas electromagnéticas son algo físico? Después de todo, no son objetos de la experiencia en ningún sentido ordinario del término; representamos estas ondas por medio de ecuaciones matemáticas bastante abstractas.

Supóngase que hay un ítem $N$ cuya naturaleza metafísica no está establecida. Ahora, supóngase que establecemos la existencia de una ley de la naturaleza que relaciona $N$ con un elemento físico paradigmático $M$. Entonces, gracias a nuestro conocimiento de esta ley, estamos en condiciones de producir o manipular $N$, explicar o predecir sus instancias, dar cuenta de sus interacciones con $M$ (y en esa medida con otros elementos físicos), etcétera, tal y como estamos en condiciones de hacerlo con las ondas electromagnéticas. Esto parece ser suficiente para sostener que $N$ tiene una naturaleza física. ${ }^{26}$

Ciertamente, si $N$ es una propiedad epifenoménica, i.e., una propiedad que es causada por propiedades físicas (o cuya instanciación es causada por un evento físico), pero que no cuenta con poder causal alguno, la línea argumentativa recién expuesta no aplica. Supuse que $N$ interactúa con propiedades físicas paradigmáticas, y para poder interactuar $N$ debe tener poderes causales. En principio, persiste entonces la posibilidad de que $N$ no tenga una naturaleza física a pesar de estar relacionada nomológicamente con propiedades físicas. Sin embargo, no se supone que las propiedades emergentes-S (y luego la conciencia si fuera una propiedad de este tipo) sean, o puedan ser, epifenoménicas. Recordemos que estas son propiedades físicas, y de acuerdo con la física contemporánea toda propiedad física tiene un perfil causal. No es el caso, por ejemplo, que las propiedades emergentes-S que tendrían los sistemas cuánticos entrelazados sean epifenoménicas. En la medida en que el dualismo naturalista considera la conciencia no solo como una propiedad que no es física sino también como

26 Nótese que esta línea argumentativa lleva a la tesis de la inestabilidad del interaccionismo, i.e., del dualismo de tipo-D en la taxonomía de Chalmers (2002). La existencia factual de interacciones nomológicas entre estados conscientes y estados físicos (que pueden incluir estados cerebrales) sería suficiente para clasificar a la conciencia como física. 
epifenoménica, hay aquí otro factor de diferenciación frente a la posición que sugiero: que la conciencia puede ser una propiedad emergente-S.

Objeción 2: Supóngase que en el mundo actual $W$ hay de hecho una ley $L$ en virtud de la cual la conciencia emerge-S. Ahora, considérese un mundo $W^{*}$ que carece de $L$, y por lo tanto de conciencia, pero que por lo demás es idéntico a $W$ en todo respecto. $W^{*}$ es concebible y sería un mundo zombi: todo elemento físico en $W^{*}$ (salvo la conciencia) tendría su contraparte en $W$.

Respuesta: Las teorías ontológicas de la emergencia, y en particular el emergentismo sobre la conciencia, usualmente conciben las propiedades emergentes como provistas de poderes causales..$^{27}$ De hecho, es usual argumentar a favor de la existencia de una propiedad emergente $E$ instanciada en una entidad $S$ sobre la base del Dictum de Alexander (Alexander, 1920): $E$ ha de estar instanciada en $S$ porque $S$ cuenta con poderes causales irreductibles al agregado de los poderes causales de los elementos físicos básicos que la integran. Luego, $W^{*}$ en realidad no es concebible: en la ausencia de $L$ diferiría de $W$ en varios respectos, a saber, a propósito de los eventos que directa o indirectamente estén relacionados con la conciencia de una manera causal.

Objeción 3: Olvidémonos del microfisicalismo. El núcleo del argumento del zombi es la idea de que existe una brecha ontológica entre los hechos físicos y las propiedades fenomenales. Las segundas no pueden derivarse de los primeros. Sostener que la conciencia puede ser una propiedad emergente-S es negar la existencia de esta brecha, puesto que la conciencia emergería exclusivamente de material físico.

Respuesta: Coincido con la idea de que las propiedades fenomenales no se siguen de hechos físicos que, ex hypothesi, no son ellos mismos fenomenales, en virtud de una necesidad metafísica (Bernal, 2012). Pero recuérdese que el emergentismo-S es una forma de fisicalismo: propone que las propiedades fenomenales son ellas mismas propiedades físicas, a pesar de ser irreductibles en términos de elementos físicos básicos. Sin duda, tenemos intuiciones dualistas fuertemente arraigadas, pero igualmente tenemos buenos motivos para creer que el

27 Ciertamente, la posibilidad de que las propiedades emergentes tengan poderes causales propios constituye el obstáculo principal que enfrentan las teorías de la emergencia correspondientes. Kim (1993; 1999; 2005) en particular ha planteado el llamado 'problema de exclusión causal'. Diversas soluciones han sido propuestas, entre ellas Loewer (2001), Shoemaker (2002), Woodward (2008), Campbell (2010), Macdonald \& Macdonald (2010). 
fisicalismo es verdadero. ${ }^{28}$ Adicionalmente, cabe anotar que parecen existir brechas ontológicas (aunque seguramente no tan agudas como en el caso de la conciencia) entre elementos microfísicos, como los átomos, y elementos físicos macroscópicos, como la rigidez, y entre fenómenos pertenecientes a niveles físicos, químicos y biológicos, como la vida y el desarrollo de procesos fisiológicos. Sin embargo, estas brechas se han cerrado o parecen susceptibles de cerrarse dentro del marco fisicalista. De hecho, algunas propuestas para cerrarlas apelan precisamente a la emergencia. ${ }^{29}$

\section{Conclusión}

El Argumento del zombi de Chalmers supone la aceptación del microfisicalismo: en pocas palabras, la tesis según la cual todo lo que existe superviene sobre los constituyentes básicos del reino de lo físico. Con base en argumentos propios de la filosofía de la física, cuestioné la veracidad del microfisicalismo y sugerí considerar en su lugar una metafísica emergentista en el sentido de la emergencia-S. Si esta metafísica resulta ser correcta, el argumento de Chalmers pierde parte de su sustento y se abre la posibilidad de que la conciencia sea una propiedad física.

\section{Agradecimientos}

Mis agradecimientos a la FMSH, por haberme otorgado la beca dentro de la cual desarrollé un trabajo del cual se derivó este texto; al Prof. Uriah Kriegel, por sus comentarios sobre un texto anterior del cual se derivó este texto; y a dos evaluadores anónimos por sus valiosas y acertadas sugerencias.

\section{Referencias}

Alexander, S. (1920). Space, Time, and Deity. London: Macmillan.

Alter, T. \& Nagasawa Y. (Eds.). (2015). Consciousness in the Physical World. Perspectives on Russellian Monism. Oxford: Oxford University Press.

Bedau, M. A. \& Humphreys, P. (Eds.). (2008). Emergence: Contemporary Readings in Philosophy and Science. Cambridge, Mass.: MIT Press.

28 Véase Papineau (2000, cap. 1).

29 Véase, e.g., Bedau et al. (2008). 
Bernal, R. (2012). E-physicalism. A Physicalist Theory of Phenomenal Consciousness. Frankfurt: Ontos Verlag/De Gruyter.

Bernal, R. (2016). An Emergentist Argument for the Impossibility of Zombie Duplicates. Paris: Working Papers Series - FMSH. <halshs-01422012v4> Disponible en: https://halshs. archives-ouvertes.fr/halshs-01422012v4

Block, N. (1995). On a confusion about the function of consciousness. Behavioral and Brain Sciences, 18, 227-247.

Campbell, J. (2010). Control Variables and Mental Causation. Proceedings of the Aristotelian Society, 110, 15-30.

Chalmers, D. (1996). The conscious mind. Oxford: Oxford University Press.

Chalmers, D. (2002). Consciousness and its Place in Nature. En S. Stich \& T. Warfield (Eds.), Blackwell Guide to the Philosophy of Mind (pp. 102-142). Oxford: Blackwell.

Chalmers, D. (2010). The Character of Consciousness. Oxford: Oxford University Press.

Chalmers, D. (2012). Constructing the World. Oxford: Oxford University Press.

Esfeld, M. (2004). Quantum entanglement and a metaphysics of relations. Studies in History and Philosophy of Modern Physics, 35(4), 601-617.

Howard, D. (2007). Reduction and emergence in the physical sciences: some lessons from the particle physics and condensed matter debate. En N. Murphy \& W. R. Stoeger (Eds.), Evolution and Emergence: Systems, Organisms, Persons (pp. 141-157). Oxford: Oxford University Press.

Humphreys, P. (2008). How Properties Emerge. En M. Bedau \& P. Humphreys (Eds.), Emergence: Contemporary Readings in Philosophy and Science (pp. 111-126). Cambridge, MA: MIT Press.

Hüttemann, A. (2004). What's wrong with microphysicalism? London: Routledge.

Kim, J. (1993). The Non-reductivist's Troubles with Mental Causation. En J. Heil \& A. Mele (eds.). Mental Causation (pp. 189-210). Oxford: Oxford University Press.

Kim. J. (1999). Making Sense of Emergence. Philosophical Studies, 95, 3-36.

Kim, J. (2005). Physicalism, or something near enough. Princeton: Princeton University Press.

Kripke, S. (1980). Naming and Necessity. Cambridge, MA: Harvard University Press.

Leuenberger, S. (2009). What is Global Supervenience? Synthese, 170(1), 115-129.

Lewis, D. (1983). New Work for a Theory of Universals. Australasian Journal of Philosophy, 61(4), 343-377.

Loewer, B. (2001). Review of J. Kim, Mind in a Physical World. Journal of Philosophy, 98(6), 315-324.

MacDonald, C. \& MacDonald, G. (Eds.) (2010). Emergence in Mind. Oxford: Oxford University Press.

McLaughlin, B. (1995). Varieties of Supervenience. En E. Savellos \& Ü. Yalcin. (Eds.), Supervenience: New Essays (pp. 16-59). Cambridge: Cambridge University Press.

McLaughlin, B. (1997). Emergence and Supervenience. Intellectica, 2, 25-43.

Nagel, T. (1974). What is it like to be a bat? Philosophical Review, 83, 435-456. 
Nida-Rümelin, M. (2007). Dualist Emergentism. En B. McLaughlin \& J. Cohen (Eds.), Contemporary Debates in Philosophy of Mind (pp. 269-286). Oxford: Blackwell.

O’Connor, T. (1994). Emergent Properties. American Philosophical Quarterly, 31, 91-104.

Papineau, D. (2000). Thinking about Consciousness. Oxford: Oxford University Press.

Papineau, D. (2008). Must a Physicalist be a Microphysicalist? En J. Hohwy \& J. Kallestrup

(Eds.), Being Reduced: New Essays on Reduction, Explanation, and Causation (pp. 126148). Oxford: Oxford University Press.

Searle, J. (1995). The Construction of Social Reality. New York: Free Press.

Shoemaker, S. (2002). Kim on Emergence. Philosophical Studies, 108, 53-63.

Freeman, A. (Ed.) (2006). Consciousness and its place in nature. Exeter: Imprint Academic.

Suppes, P. (2002). Representation and Invariance of Scientific Structures. Stanford, CA: CSLI Publications.

Teller, P. (1986). Relational Holism and Quantum Mechanics. Brit. J. Phil. Sci., 37, 71-81.

Van Cleve, J. (1990). Mind-Dust or Magic? Panpsychism Versus Emergence. Philosophical Perspectives, 4, 215-226.

Woodward, J. (2008). Mental Causation and Neural Mechanisms. En J. Hohwy and J. Kallestrup (Eds.), Being Reduced: New Essays on Reduction, Explanation, and Causation (pp. 218262). Oxford: Oxford University Press. 